\section{Prescriber focus}

Je suis consterné et révolté de découvrir l'enquête conjointe de la FMH et de l'IHA-IMS: «Prescriber focus».

Pour qui vous prenez-vous, pour qui vous prenez-nous? La FMH n'a pas à favoriser les enquêtes de marketing parmi ses membres: ouvrir nos dossiers et informer sur nos habitudes de pratiques, c'est aider les marchands de médicament à mieux nous manipuler. La publicité n'est pas de l'information scientifique. Cela ne m'intéresse pas d'expliquer aux délégués médicaux comment il faut me caresser le poil pour influencer ma prescription.

Il serait possible d'enquêter utilement pour améliorer la qualité et l'économicité des traitements, d'enquêter afin d'améliorer l'information et la formation des praticiens.

Vous abusez de notre temps en nous demandant des statistiques détaillés sur notre comportement par exemple avec neuf IEC (deux génériques, pourquoi pas les autres: parce que leurs firmes ne cotisent pas chez IHA-IMS?). Est-ce dans ces détails que réside la qualité de notre pratique? Le comble: votre petit cadeau; et la honte suprême: le promesse de récompense à «l'équipe du cabinet» qui aura convaincu son médecin de remplir le questionnaire.

Croyez-nous que nous nous amusons avec cette joyeuse émulation? A vomir!

Je demande à «ma» FMH de déclarer précisément:

- si et combien elle reçoit en jouant le cheval de Troie au service de l'industrie;

- si et combien de moyens (temps, salaire, envoi de courrier) elle a consacré à cette demande totalement déplacée.

Enfin je demande à mes confrères de se joindre à ma protestation, de refuser de remplir ce questionnaire, et s'ils l'ont rempli de demander, comme promis au bas du formulaire, l'effacement de leurs données «à la date possible la plus proche».

Je me réjouis de collaborer avec la FMH (ou avec l'AMI Association des médecins indépendants indépendants des financements de l'industrie) à des recherches ou à des enquêtes correspondant à notre éthique médicale.

Dr H. Burkhalter, Lausanne

\section{Replik}

Zum Fragebogen halte ich folgende Punkte fest:

1. Die FMH erhält aus dieser Befragung nur anonymisierte Daten.

2. Wer nicht will, dass seine Daten weitergegeben werden, kann dies auf dem Fragebogen markieren.

3. Die Dignitätsdatenbank hat mit dieser Umfrage weder inhaltlich, formal, noch operativ etwas zu tun. Ich halte fest, dass die Vertraulichkeit bis jetzt absolut gewahrt werden konnte und verweise dabei auf die entsprechenden Regelungen.

4. Incentives sind in solchen Studien üblich. $\mathrm{Ob}$ der einzelne mitmachen will, ist seine eigene Entscheidung.

Dr. med. H. H. Brunner, Präsident FMH

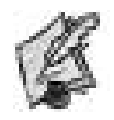

\section{Letzte TARMED-Installationen}

Die Weihnachts- und Neujahrstage 2003 werden mir in unvergesslicher Erinnerung bleiben! Die freien Tage oder Stunden konnten endlich einmal beruflich voll genutzt werden, man fand im Festtagstrubel Zeit, den letzten Schliff an der TARMED-Installation auf dem PC zu vollenden. Oder eben doch noch nicht ganz zu vollenden, dafür zu erkennen, dass noch irgendwo irgendetwas nicht ganz genau so geht, wie man glaubte, es würde gehen. Und das Tollste an der TARMEDGeschichte ist, dass sie selbst nach über 10jährigen Geburtswehen nicht fehlerfrei ist, dass man immer wieder über Unklarheiten stolpert, dass man trotz aller TARMED-Seminarien und Leitfäden doch recht allein ist, die grosse Standesorganisation (FMH = für meine Hilfe) hilft mir im Detail eben nicht und ich gewinne das Gefühl, dass auch dieser an und für sich interessante und moderne Zeittarif wieder jede Menge Interpretationen offenlassen wird, dass es wieder viel eigene Phantasie braucht, damit man sich nicht zu schlecht bewertet oder neue, nun essentielle Nummern vergisst. Ich befürchte, dass selbst dieser Tarif, der in der ganzen Schweiz identisch sein soll, dies mitnichten ist, dass es jede Menge Tricks geben wird, welche eine Angina auf viele Arten abrechnen lässt, etwas ehrlich (beschränkt) oder eben eher gerissen und tarifausschöpfend. Mit grosser Selbstverständ- 
lichkeit wird verlangt, dass die Leistungserfassung ab 1. Januar 2004 nach TARMED zu erfolgen hat, ob die kontrollierenden Kassen dies auch ab 1. Januar 2004 sicher und fehlerfrei können, ist bis dato offen.

Noch ein Gedanke: Soll ich die TARMED-Installationsstunden als Fortbildung verbuchen (Selbststudium)?

Ich wünsche uns allen Zuversicht und starke Nerven, dass wir im Jahr 1 nach TARMED-Geburt den Glauben an eine gerechte Tarifierung nicht verlieren.

R. H. Schütz, Burgdorf

\section{Replik}

Die Verhandlungen vom September 1999 haben bei den Versicherern leider kein positives Echo gefunden, so dass TARMED, wie er jetzt in Kraft getreten ist, offiziell kein «Splitting» der technischen Leistung vorsieht. Im betreffenden Artikel wird allerdings die Möglichkeit von individuellen Übereinkommen zwischen den Spitälern und den Belegärzten erwähnt. So wird zweifellos auf der Basis von solchen Modellen, die von Fall zu Fall entwickelt werden müssen, eine Lösung des Problems, wie die technische Leistung aufgeteilt werden soll, gesucht werden müssen.

Jacques-Henri Weidmann, Tarifdienst FMH

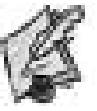

Information zum Anwendungsbereich von TARMED per 1. Januar 2004 im UV-/MV-/ IV-Bereich

Mit Bestürzung habe ich vom obgenannten Artikel Kenntnis genommen, der so nicht korrekt ist. Anlässlich der Abschlussverhandlungen des TARMED-Kapitels Anästhesie und Reanimation am 21. September 1999 in Luzern wurde unter Antrag 8 «Berufsnebenkosten» klar festgehalten, dass die TL in den Positionen der perioperativen Betreuung (Leistungen 26.0035ff entsprechend heute 28.00010ff in TARMED 1.1r) «derjenigen Person bzw. Institution zukommt, welche die damit verbundenen Kosten trägt» (Zitat Protokoll 21.9.1999)

Der Kostenträger ist entweder das Spital oder der Belegarzt. Die in dieser Position subsumierten Kosten sind u.a. Verbandsbeiträge, Fortbildung, Telekommunikation, Miete, Personalkosten, Berufshaftpflicht und Versicherungsprämien, etc. Im erwähnten Artikel wird die Rechnungsstellung und damit auch der Besitzstand dieser TL klar dem Spital zugewiesen. Der am Schluss des Artikels lapidare Satz «vorbehalten bleiben individuelle Vereinbarungen zwischen dem Belegarzt und dem Belegarztspital» darf nicht darüber hinwegtäuschen, dass diese TL korrekt und vertragsgemäss dem Kostenträger zuzuweisen ist und keiner wie auch immer gearteten Vereinbarung bedarf. Es kann und darf nicht Aufgabe von über 70 Anästhesieabteilungen an Belegarztkliniken sein, diese TL von der Klinik einzufordern. Als Verhandlungsträger der SGAR an dieser Sitzung bitte ich um Klarstellung dieses Sachverhaltes in der SÄZ.

Dr. med. X. Troxler-Mattmann, Luzern

\section{Les rapports entre les pharmaciens et le corps médical}

Je désirerais avoir votre opinion sur un point quelque peu choquant pour ma part concernant les rapports que nos «confrères» pharmaciens désirent (encore?) entretenir avec le corps médical. J'illustre mon propos par l'anecdote suivante: pas plus tard qu'hier, dans une pharmacie d'une certaine importance, en l'occurrence la Pharmacie Principale du centre Balexert de Genève, je désire me procurer un emballage de 16 comprimés de Co-Dafalgan pour mon propre usage. Après avoir prouvé mon appartenance au corps médical et que l'emballage me soit apporté, quelle n'est pas ma surprise en voyant le montant et le libellé du ticket de caisse. En effet, pour une boîte coûtant Fr. 7.05 sur laquelle on me fait «généreusement» un rabais de 10\% (soit 70 cts, que je ne demandais pas), je vois rallonger le montant de Fr. 4.30 pour une «validation d'ordonnance» (laquelle?!) et de Fr. 7.55 pour un «suivi thérapeutique», soit au total un surplus de Fr. 11.85 (soit $168 \%$ du prix initial!!). Je me demande de qui l'on se moque lorsque l'on voit la petite phrase annotée au bas du ticket: «Construisons ensemble votre avenir santé»! Je pense qu'en ce qui concerne ma santé je peux fort bien me passer des conseils prodigués par une assistante en pharmacie (il n'y en a heureusement pas eu besoin). Je ne crois pas qu'un tel manque de bons échanges puisse favoriser nos rapports avec le monde des pharmaciens suisses. Pour ma part je me le tiens pour dit et trouverai d'autres filières pour me procurer des médicaments nécessitant un tel «suivi thérapeutique».

Dr S. Bosquet, Genève 